\title{
Optical Core Networks Research in the e-Photon-ONe+ Project
}

\author{
Franco Callegati, Member, IEEE, Filippo Cugini, Paul Ghobril, Sebastian Gunreben, \\ Víctor López, Student Member, IEEE, Barbara Martini, Member, IEEE, Pablo Pavón-Mariño, Member, IEEE, \\ Marcell Perényi, Namik Sengezer, Student Member, IEEE, Dimitri Staessens, János Szigeti, and \\ Massimo Tornatore, Member, IEEE
}

\begin{abstract}
This paper reports a summary of the joint research activities on Optical Core Networks within the e-Photon-ONe+ project. It provides a reasonable overview of the topics considered of interest by the European research community and supports the idea of building joint research activities that can leverage on the expertise of different research groups.
\end{abstract}

Index Terms-Congestion resolution, GMPLS, optical networks, optical packet switching, physical impairment, protection, restoration, service oriented networks, traffic engineering, wavelength routing.

\section{INTRODUCTION}

$\mathbf{E}$ photon/one was a Network of Excellence ( $N o E$ ) funded by the European Commission (EC) in the context of the 6th Framework Programme (FP6) with the primary goal of fostering the integration of European research institutions active in optical networking research [1], [2].

e-Photon-ONe was a large project, involving about 40 institutions and 500 researchers. It was funded for two

Manuscript received August 03, 2008; revised November 15, 2008, March 20, 2009, and May 14, 2009. First published May 29, 2009; current version published August 28, 2009. This work was supported by the e-Photon/ONe+ and BONE ("Building the Future Optical Network in Europe") projects funded by the European Commission through the 6th and 7th ICT-Framework Programme.

F. Callegati is with the Department of Electronics, Computer Sciences and Systems, University of Bologna, Italy (e-mail: franco.callegati@unibo.it).

F. Cugini and B. Martini are with CNIT, Pisa, Italy (e-mail: filippo. cugini@cnit.it; barbara.martini@cnit.it).

P. Ghobril was with Orange Labs, Lannion, France. He is now with Envergus, Lannion, France (e-mail: paul.ghobril@orange.fr).

$\mathrm{S}$. Gunreben is with the Institute of Communication Networks and Computer Engineering, University of Stuttgart, Germany (e-mail: sebastian.gunreben@ikr.uni-stuttgart.de).

V. López is with the Departamento de Ingeniería Informática, E.P.S., Universidad Autónoma de Madrid, Spain (e-mail: victor.lopez@uam.es).

P. Pavón-Mariño is with the Department of Information Technologies and Communications, Polytechnic University of Cartagena (UPCT), Cartagena, Spain (e-mail: pablo.pavon@upct.es).

M. Perényi and J. Szigeti are with the HSNLab, Department of Telecommunications and Media Informatics (TMIT), Budapest University of Technology and Economics (BME), Hungary (e-mail: perenyim@tmit.bme.hu; szigeti@tmit.bme.hu).

N. Sengezer is with the Department of Electrical and Electronics Engineering, Bilkent University, Ankara, Turkey (e-mail: namik@ee.bilkent.edu.tr).

D. Staessens is with Department of Information Technology (INTEC), Ghent University-IBBT, Belgium (e-mail: dimitri.staessens@intec.ugent.be).

M. Tornatore is with the Dipartimento di Elettronica e Informazione (DEI), Politecnico di Milano, Italy (e-mail: tornator@elet.polimi.it).

Color versions of one or more of the figures in this paper are available online at http://ieeexplore.ieee.org.

Digital Object Identifier 10.1109/JLT.2009.2024090 years (2004-2005) and as it proved successful, for two more years (2006-2007) under the e-Photon-ONe+ name. The e-Photon-ONe community currently supports the BONE project (http://www.ict-bone.eu) that stemmed from the previous experience. The e-Photon-ONe consortium gained worldwide visibility and reputation. An example is the co-sponsorship (with COST and NSF) of the "US/EU Workshop on Key Issues and Grand Challenges in Optical Networking" [3].

The size of the project raised significant problems of management. The concept of Virtual Departments (VDs) was defined as the container and promoter of activities aimed at achieving durable integration, i.e., to promote joint research activities $(J A s)$, identify new research topics, etc..

This work reports the main results of the activities developed by the Virtual Department on optical core networks and technologies $(V D-C)$ in the last two years of the project (results of the previous period are summarized in [4]). The complete list of JAs in VD-C is presented in Table I with list of participants.

The rest of the paper is organized as follows. Section II provides an overview of the reference network scenario. Then a summary of selected JAs is reported organized per topic: traffic engineering in Section III, network resilience in Section IV, optical packet switching in Section V, and finally service oriented optical core networks in Section VI.

\section{REFERENCE SCENARIO}

The typical Optical Core Network (OCN) architecture comprise a data plane (DP) and a control plane (CP). The former is responsible for user data flow forwarding and here is assumed that it mainly exploits all-optical switching. The latter is responsible for the logical networking functions, e.g., routing, resilience and management.

Alternatives for multiplexing and switching in the DP range over a wide set of alternatives, providing different trade-offs between flexibility and complexity; from fibre and wavelength switching to sub-wavelength switching (optical time division multiplexing, OTDM, optical burst switching, OBS, optical packet switching, OPS)

Because of the large traffic flows carried by the OCNs, critical issues for the $\mathrm{CP}$ are reliability and network survivability as well as traffic engineering and contention resolution. GMPLS offers capabilities able to address many of these issues and is a major candidate for OCNs' CP.

New topics are also emerging when considering future mass market, bandwidth-greedy applications, such as Grid Computing and Service Delivery Platform, requiring "on demand" 
TABLE I

JOINT ACTIVITIES IN e-Photon-ONe+ VD-C

\begin{tabular}{|c|c|}
\hline Activity Title & Participants \\
\hline \multicolumn{2}{|l|}{ Traffic engineering } \\
\hline $\begin{array}{l}\text { Regular Reconfiguration of Light-Trees in Multi-layer Optical Networks } \\
\text { A Comparative Study of Single-layer and Multi-layer Traffic Engineering Strategies } \\
\text { Implementation and experimental verification of a multi-layer integrated routing scheme for traffic } \\
\text { engineering } \\
\text { Dynamic optical circuit-switched transport networks }\end{array}$ & $\begin{array}{l}\text { M. Perényi P. Soproni, T. Cinkler } \\
\text { N. Sengezer B. Puype, E. Karasan, M. Pickavet } \\
\text { S. Gunreben F. Agraz, S. Spadaro } \\
\text { P. Ghobril E. Le Rouzic, H. Nakajima, R. Watza, } \\
\text { J. Rzasa, W. Kabacinski, S. Hanczewski, S. } \\
\text { Spadaro }\end{array}$ \\
\hline \multicolumn{2}{|l|}{ Network Resilience } \\
\hline $\begin{array}{l}p \text {-Cycle Protection in Multi-Domain Environment } \\
\text { Survivability in Multidomain networks: applicability and signaling } \\
\text { Effects of Outdated Information on Protected Routing in WDM Networks } \\
\text { QoT-aware control plane }\end{array}$ & $\begin{array}{l}\text { J. Szigeti R. Romeral, D. Larrabeiti, T. Cinkler } \\
\text { D. Staessens R. Romeral } \\
\text { Massimo Tornatore A. Pattavina, R. Munoz, R. } \\
\text { Casellas, R. Martinez } \\
\text { F. Cugini C. Pinart, I. Martinez, E. Le Rouzic, M. } \\
\text { J. Poirrier, P. Castoldi, L. Valcarenghi, N. Sambo, } \\
\text { N. Andriolli, A. Giorgetti }\end{array}$ \\
\hline \multicolumn{2}{|l|}{ Optical Packet Switching } \\
\hline $\begin{array}{l}\text { Key Parameters for Congestion Resolution in OPS/OBS Networks } \\
\text { Comparison of end-to-end packet ordering issues in synchronous and asynchronous OPS networks }\end{array}$ & $\begin{array}{l}\text { F. Callegati W. Cerroni, L. H. Bonani, G. S. } \\
\text { Pavani } \\
\text { P. P. Mariño J. Veiga Gontán, J. García Haro, D. } \\
\text { Careglio, M. Klinkowski, J. Solé-Pareta }\end{array}$ \\
\hline \multicolumn{2}{|l|}{ Service Oriented OCNs } \\
\hline Multilayer Switching Algorithm for an All-Optical Router & $\begin{array}{l}\text { B. Martini V. Martini, F. Baroncelli, P. Castoldi, } \\
\text { L. Rea, C. Zema, S. Pompei, F. Matera, L. } \\
\text { Wosinska, H. Su, A. Valenti } \\
\text { V. López J. A. Hernández, J. Aracil, J. P. Fernán- } \\
\text { dez Palacios, O. González de Dios }\end{array}$ \\
\hline
\end{tabular}

network services with configurable bandwidth, availability, end-to-end delay, etc. Service architectures have been defined by the principal standardization bodies, such as the IP Multimedia Subsystem (IMS) by 3GPP [5] and the Next Generation Network (NGN) by ITU-T [6]. Unfortunately none of them foresee any exploitation of the Generalized Multi Protocol Label Switching (GMPLS) CP capabilities. This opens a whole set of new problems that are particularly important for OCNs.

All these issues were addressed at some extent by VD-C, with the sole exception of Optical Burst Switching. A separate workpackage, working in collaboration with VD-C, was devoted to this topic but formally reported results separately. A summary of the activities on OBS can be found for instance in [7].

\section{TRAFFIC ENGINEERING}

Four joint activities addressed traffic engineering mainly focusing on multilayer (ML) networks. The first activity concerned multicast in ML networks and showed the benefit of reconfiguring the multicast trees periodically to cope with traffic variations [8], [9]. The second activity compared traffic engineering (TE) strategies in ML networks. TE may be implemented in the upper electronic layer only, or in both electronic and optical layer with periodic topology reconfigurations of the latter. Results showed the benefit of traffic flow reconfiguration in comparison to a statically configured network [10]. The third activity focused on the realization issues of a traffic engineering algorithm for ML optical networks which is implemented in an optical test-bed [11]. The last activity had a slightly different focus. It assumed a network with different switching granularities and proposed a comparison framework to assess the advantages/drawbacks of using a dynamic switching technology (for instance OBS) with respect to a more coarse wavelength switching [12], [13].

\section{A. Regular Reconfiguration of Light-Trees in Multilayer Optical Networks}

Multicast (MC) applications will likely increase in the future Internet [14] and network engineering suggests implementing multicast delivery in the lowest layers of the network to avoid waste of bandwidth due to unicast-based distribution of MC flows [8], [15], [16].

The problem considered here that of dynamic multicast (MC) trees, where the members are continually changing, causing a degradation of the tree as it diverges from the optimum. Regular reconfiguration of the $\mathrm{MC}$ tree can solve this degradation, but it also has drawbacks: computation effort, short disruption in the data transmission flow, additional signalling overhead. Therefore, it is important to understand the cost/benefit trade-off of reconfiguring the $\mathrm{MC}$ tree.

The reference scenario is a two-layer network, where the upper electronic layer is packet switching capable, while the lower, optical layer is wavelength switching capable. ${ }^{1}$ The traffic consists of dynamic, multicast delivery demands, for instance a digital media distribution service, where the audience is varying in time.

\footnotetext{
${ }^{1}$ The electronic layer can perform traffic grooming. The control plane has information about both layers and both layers are involved in routing.
} 


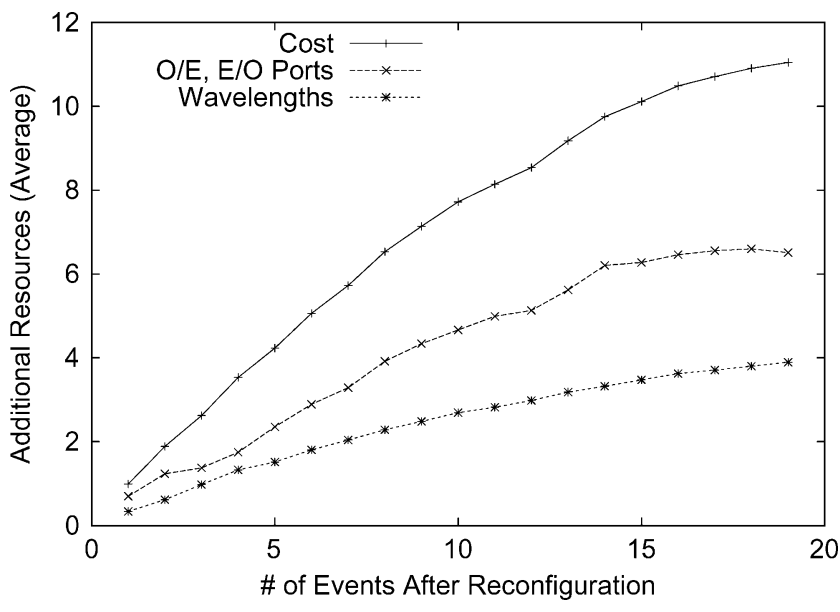

Fig. 1. Average additional cost of routing after reconfiguration.

The optimal design of the MC tree can be solved by using Integer Linear Programming (ILP) [8]. Unfortunately the exact solution is NP-complete, and heuristic algorithms can be defined to make the computation more efficient [16]. Obviously these solutions depend on the distribution of the members of the tree. When members leave or enter the multicast tree an good solution is applicable only with a reconfiguration of existing paths.

The alternative to reconfiguration is to modify the tree without reconfiguring it completely. Heuristics were defined to this end, presented in [9]. The results presented here refers to the Accumulative shortest path (ASP) heuristic, that simply connects newly arriving endpoints to the MC tree applying Dijsktra's algorithm, and clears branches leading to departed endpoints.

The results refer to the COST 266 European reference network [17]. The ILP problem was solved using the CPLEX optimizer. The number of wavelengths per link was 8 . Lightpaths can be routed up to the electronic layer to perform (tree-) branching in any node. However, O/E (optical to electronic) and $\mathrm{E} / \mathrm{O}$ conversion was assumed to be twice more expensive than switching in the optical layer.

It is assumed that the tree is periodically re-configured. The arrival of a new demand or the departure of an existing one is called an event. Fig. 1 shows the average additional cost increase of the multicast tree as a function of the number of elapsed events after reconfiguration, split in routing cost, number of $\mathrm{O} / \mathrm{E}, \mathrm{E} / \mathrm{O}$ conversion ports and number of wavelengths used. As expected the more the events after reconfiguration the more the $\mathrm{MC}$ tree diverges from the optimal and the greater the additional cost.

The interesting result is that, if reconfiguration is implemented, it is possible to identify an optimal length of the reconfiguration period, if we take into account the negative aspects of reconfiguration as a penalty (see Fig. 2). Our further results show that the reconfiguration seems to be especially useful if grooming is not possible. Still, a number of technical challenges must be addressed to make reconfiguration practical, like the seamless switchover of traffic from the old to the new tree.

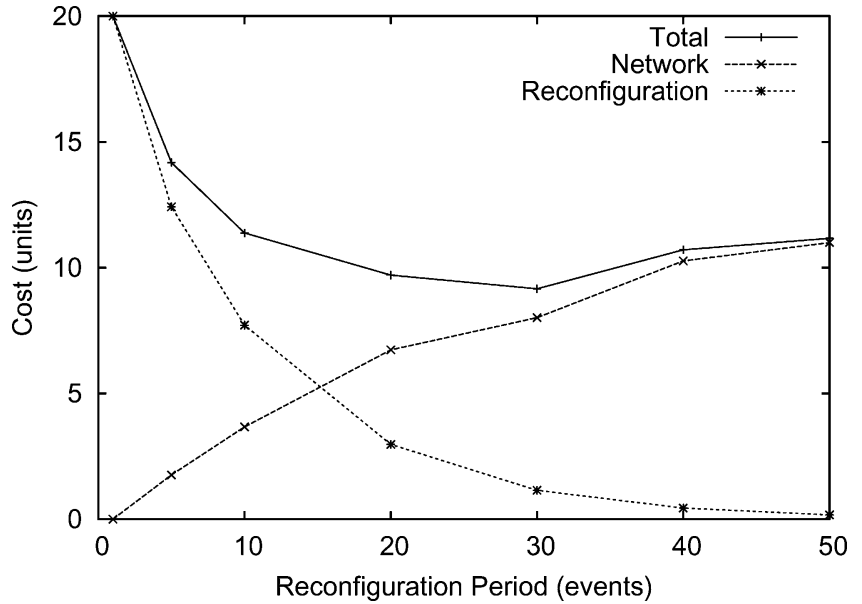

Fig. 2. Total, network, and reconfiguration costs as a function of the reconfiguration period. The minimum of the total cost curve suggests the optimal length of the reconfiguration period.

\section{B. Implementation and Experimental Verification of a Multilayer Integrated Routing Scheme for Traffic Engineering}

This research activity studied a multilayer routing algorithm and its real life implementation in a test-bed including an electronic layer (SDH) on top of an optical transport layer (WDM) with a common CP based on GMPLS.

The CARISMA test-bed [11] is essentially a wavelength-routed optical network. It sets-up end-to-end connections as lightpaths and also allows the set-up of finer grain connections thanks to the Forwarding Adjacency (FA) concept. It permits the aggregation of higher-order LSPs into these lower-order ones. The routing protocol advertises these lower-order LSPs as FA-LSPs. The nodes may use FA-LSP for path computation, nesting lower-order LSPs into FA-LSPs [18]. The FA functionality was implemented with a proprietary extension to the routing protocol.

The TE algorithm implemented is called Weighted Integrated Routing (WIR) and was proposed in [19]. It assumes a co-location of the electrical and optical nodes and operates in a two-step approach: the first step includes a shortest path search from source to destination; the second step chooses a subset of intermediate nodes and checks the feasibility of a path using these nodes. The optimal path is chosen according to the cost, including number of wavelength conversions, number of electrical or optical hops or link occupancy.

The implementation of the WIR faced some restrictions imposed by the test-bed.

- Wavelength continuity: GMPLS does not support it. The test-bed implementation includes this information using a nonstandard OSPF-TE extension.

- Number of optical hops: The FA does not provide any information of the underlying optical links and hides the number of optical nodes in the path to the electronic layer.

- Cost metrics: The cost metrics available are limited; here were used the number of electrical and of optical hops (if available). 


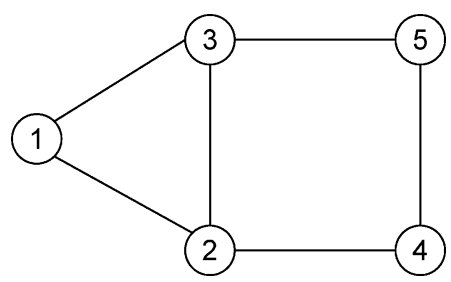

Fig. 3. Network topology used for simulation. The links are uni-directional, carrying two wavelengths with STM-16 capacity.

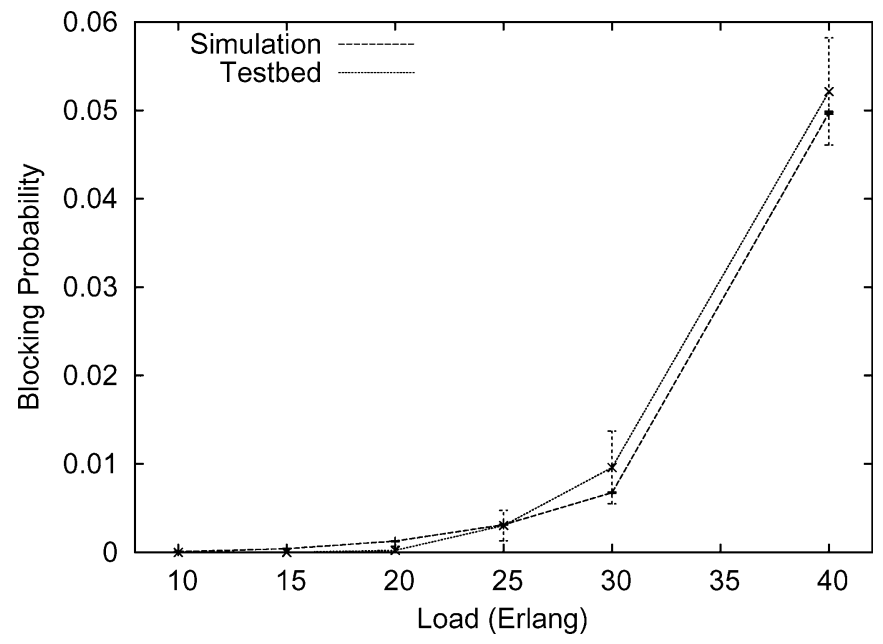

Fig. 4. Comparison of the connection blocking probability.

The WIR algorithm was tested in the optical test-bed. Its performance was also evaluated by simulations in a simple network (Fig. 3). In the simulation, the $\mathrm{CP}$ advertises the link capacity with STM-4 granularity. The traffic demands request STM-4 bandwidth and are uniformly distributed.

Fig. 4 shows the connection blocking probability comparing test-bed and simulation results. Over the considered load range, measurement and simulation fit well.

The outcome of this activity showed that the ML routing algorithm is in principle realizable in a real network, although some limitations were either solved by proprietary protocol extensions or skipped in the implementation. In particular the implementation required the extension of the GMPLS CP.

\section{NETWORK RESILIENCE}

Network resilience in OCNs has been widely investigated in the past, nonetheless, some of the recent progresses pose new challenges. It was already mentioned that OCNs will likely interconnect several optical domains. It is reasonable to assume that the end-users' expectation is that they get the same or near the same reliability for interdomain as for intradomain connections. Therefore, protection and restoration in multidomain scenarios is a key issue that was investigated by two JAs [20]-[22].

Furthermore, the emerging applications call for an intelligent optical network control plane, such as that provided by GMPLS. Unfortunately the control plane may impact on the network performance, and most of the studies on dynamic traffic routing tend to neglect the effect of control plane on routing performance. The JA summarized in Section IV-A tackle this problem [23].

Similarly, while propagating through the optical network, a signal may degrade in quality as it encounters physical impairments, and this may in turn make the Bit Error Rate (BER) at the destination unacceptably high. Thus, Quality of Transmission (QoT) parameters have to be introduced in the GMPLS protocol suite to provide reliable lightpath provisioning. The last JA investigates these issues [24], [25].

\section{A. Effects of Outdated Information on Protected Routing in WDM Networks}

This activity concentrated on the general effects of outdated information in a control-plane enabled optical network, a problem not investigated to date. In distributed-GMPLS networks, each node builds a network image to identify the best path to route a connection (source routing).

As an effect of control delays, this image may not be updated and routing not optimized. The information to build this image depends on various factors, e.g., which protection is applied and whether wavelength conversion is enabled. Moreover, in the case of shared protection, the state of shareable backup resources has to be disseminated and ad-hoc routing protocol (such as OSPF-TE) extensions may be needed [26].

The contributions to the control delay can be summarized as: information propagation delay, set-up delay, switching delay, processing delay, periodical database update. It was proposed to evaluate them using a simple delay model based on the following assumptions.

1) Constant Control Delay: The control delay is fixed and equal to $\tau$.

2) Negligible Set-Up Time: Once routing has been identified, provisioning occurs without no delays.

3) Identical network vision: All the nodes share the same network vision, referred to the instant $t-\tau$.

Our simplified approach enables the effect of a wide range of control delay values to be quantified, independent of the specific routing/control algorithms and parameters (update frequency, amount of information).

In summary, in the JA the control-delay effects on routing performance were analyzed [23], using realistic case-study network topologies in a dynamic network environment. The study considered a network without protection, with dedicated path protection (DPP) and with shared path protection (SPP) with (VWP) and without (WP) wavelength conversion. The considered metric is the blocking probability (BP).

Fig. 5 shows the BP for the DPP and SPP case (under WP and VWP assumption). Curves are plotted as a function of $\tau$, considering 100 arrivals per second (which leads to a network load of around 0.55 ). The delay on the $x$-axis is a relative measure, expressed as the ratio between the absolute delay $D$ and the average holding time $H T$. These curves show three distinct phases.

- Phase 1-Not influential delay: In this first phase, the BP is constant and it is not influenced by the delay. 


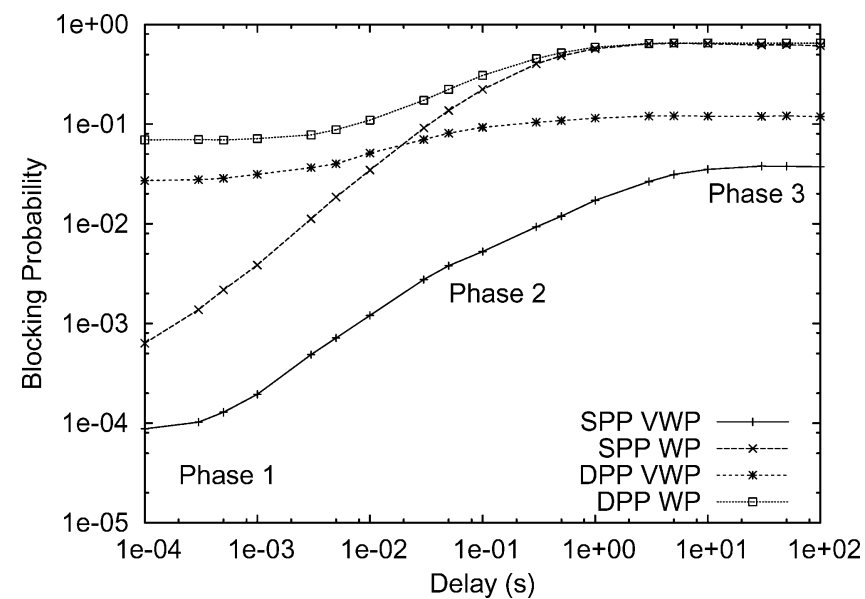

Fig. 5. Total blocking probability for DPP and SPP routing in VWP and WP case.

- Phase 2-Linear increase: Outdated information starts affecting the quality of source-routing, causing a significant and linear increase of the BP.

- Phase 3-saturation: The BP is not affected by increases in the control delay, since the network image at the source node is now uncorrelated to the actual network state.

As a matter of fact, the provisioning of a connection over a given path in the VWP case fails only in the case where all the channels on a link are saturated, while in the WP case only the chosen channel has to be free to allow a successful provisioning of the connection over the chosen path.

\section{B. QoT-Aware Control Plane}

The set up of transparent connections (T, i.e., lightpaths) or nonfully transparent connections (NoT, i.e., lightpaths with some intermediate nodes performing opto-electronic regeneration), requires the enhancement of the GMPLS protocol suite to include information related to both Quality of Transmission (QoT) and to the presence of shared-per-node regenerators [27]. In this study, the Signalling Approach (SA)-based GMPLS enhancement proposed in [24] is considered.

In $\mathrm{SA}$, no extensions are introduced in the routing protocol which calculates routes ignoring QoT. Then, SA performs the dynamic estimation of the QoT during the signalling phase by collecting QoT parameters from intermediate nodes. At the destination node, if the accumulated information is within an acceptable range, the lightpath set-up request is accepted. Otherwise the lightpath request is rejected and further set up attempts following possibly link-disjoint routes are triggered.

The main advantage of SA is that it avoids the flooding of QoT parameters and regenerator availability and preserves control plane scalability. However, it may increase the amount of control plane packets and delay the lightpath establishment process. Expanding upon [24], this activity evaluated the performance of the SA when both QoT and shared regenerator information are considered. The performance is evaluated by means of a custom built $\mathrm{C}++$ event-driven network simulator. A Pan-European topology with 17 nodes and 32 links is considered [25]. Each link carries 40 wavelengths. Each network node

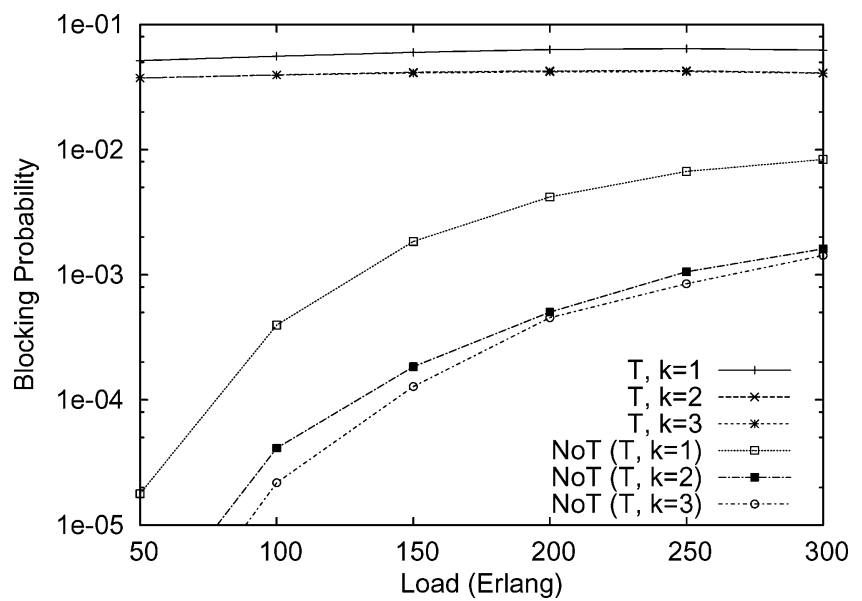

Fig. 6. Blocking probability within $\mathrm{k}$ (transparent) set up attempts and after the successive set up attempt exploiting $N$ regenerators per node. (a) $k=1$; (b) $k=2$; (c) $k=3$.

is equipped with $\mathrm{N}$ shared-per-node regenerators. Connection requests are dynamically generated with uniform distribution among all node pairs. Network load is kept limited in order to experience connection blocking due mainly to unacceptable QoT or lack of regenerators.

Fig. 6 shows the blocking probability (BP) of T connections within $k$ set up attempts ( $k$ equal to 1,2 and 3 ). In addition it shows the BP of the first NoT set up attempt performed upon the unsuccessful $k$-th transparent set up attempt. In this example the nodes are equipped with $N=2$ shared-per-node regenerators. Results show that, by exploiting successive set up attempts, the overall BP of transparent connections decreases. In addition, also the NoT connection BP, due to the lack of regenerators, decreases with the increase of the number $k$ of transparent connection set up attempts. Indeed, a higher number of explored routes and nodes allows a saving in regenerators and improves the likelihood of establishing NoT connections. However, while the increase from $k=1$ to $k=2$ leads to significant $\mathrm{BP}$ reductions, increasing $k$ from 2 to 3 provides negligible reductions. Thus, just two set up attempts before resorting to regenerators guarantee the best performance.

\section{Contention Resolution Strategies in Optical PACKET SWITCHING}

The focus of the research on OPS was on solving contention by the use of scheduling algorithms exploiting wavelength conversion and delay lines in a combined way.

In the former activity a new comparison metric is defined that allows to look at the complexity/performance trade-off of different scheduling alternatives under a new perspective [28], [29]. The latter activity explores the issue of designing scheduling algorithms that are able to maintain the packet sequence and, therefore, may be suitable for QoS sensitive traffic [31], [32].

Both activities referred to an OPS switching system able to emulate output queuing with delay lines and converters shared per output port. The $N$ input/output ports are equipped with $F$ fibres each, carrying $W$ wavelengths and with $B$ delay lines. Consequently the number of input/output channels per node is 
$N \cdot F \cdot W$. As usually in these studies, the focus is on the packet loss probability (PLP).

The JA here reported focused on contention resolution algorithms that exploit the wavelength and time domains in a coordinated manner. This is called Channel and Delay Selection (CDS) scheduling. Several algorithms were proposed to solve this problem but a general framework to compare different alternatives was missing. We defined the concept of scheduling space and proposed to use it as the basis to assess complexity/ performance trade-offs.

Given an arriving packet that will be forwarded to a given output port, the CDS algorithms must choose on which of the $C \leq F \cdot W$ channels potentially available on that port the packet will be transmitted. The scheduling space is the set of choices

$$
s(i, j, k)=\left(t_{i}, j, k\right) \in \mathcal{S}
$$

where $i$ gives the delay out of the $B$ available per channel, $j$ gives the wavelength and $k$ the fiber. The number of elements (i.e., the cardinality) in $\mathcal{S}$ is $|\mathcal{S}|=B \cdot C$.

In an ideal switching matrix with full range wavelength conversion (FWC), $|\mathcal{S}|=\left|\mathcal{S}_{\mathrm{FWC}}\right|=B \cdot W \cdot F$. However, in real systems $|\mathcal{S}| \leq\left|\mathcal{S}_{\mathrm{FWC}}\right|$ as a result of hardware or software limitations. For instance, if the switching matrix is equipped with limited range wavelength converters (LWC), the $W$ wavelengths per fiber are divided in wavebands of $L$ wavelengths and conversion may happen only within the same waveband, therefore $|\mathcal{S}|=B \cdot L \cdot F \leq\left|\mathcal{S}_{\mathrm{FWC}}\right|$.

We believe $|\mathcal{S}|$ is a measure of the cost of the CDS algorithm, since it is correlated to the amount and kind of devices needed to implement the switching matrix, and a fair comparison between scheduling algorithms must be done with the same values of $|\mathcal{S}|$. The engineer has to dimension the $B, F, W(L)$ parameters.

For instance it is known that, in general, it is more profitable to invest in channels rather than in delays [30]. But when we compare keeping $|\mathcal{S}|$ fixed we discover that the best performance is obtained with 32 wavelengths and 1 delay per interface, and not with 64 wavelengths and no delays as known results would suggest.

Another nonintuitive result is that LWC is not necessarily worse than FWC. Comparing FWC and LWC with the same scheduling space it happens that a small increase in delays may well compensate the limited range conversion, whilst also improving the overall performance as shown by the example in Fig. 7 [28].

\section{SERVICE ORIENTED OCNs}

To provide advanced and QoS-enabled connectivity services, to new IT application such as Global Grid Computing, the OCNs must be enhanced with the capability to interact with the applications and consistently perform the network resource allocation. The problems in this field were:

- the support for direct invocation and fulfilment of QoSenabled connectivity services [33], [35];

- the implementation of decision algorithms to share the resources among the incoming service and map the traffic flows on the network resources [36].

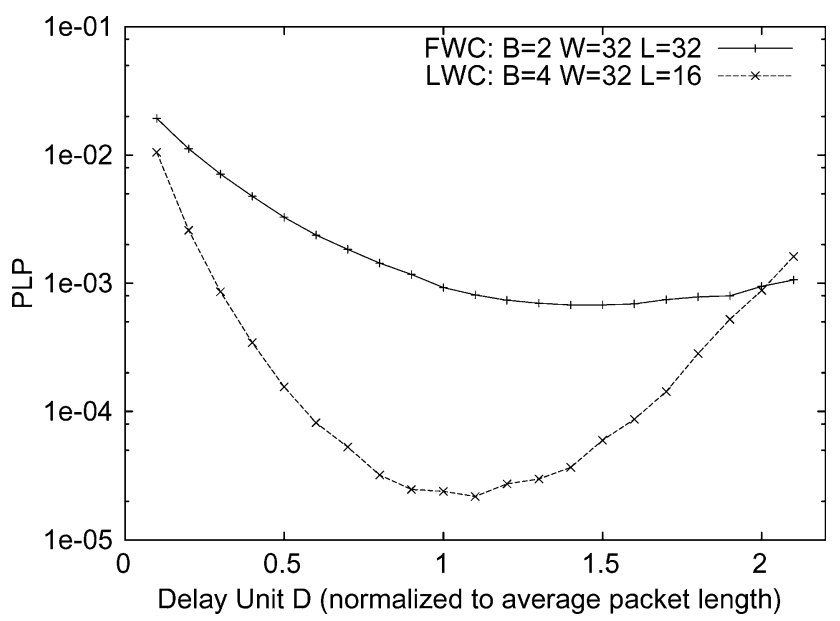

Fig. 7. PLP as a function of the delay unit $D$ comparing FWC and LWC, for a switch with $\left|\mathcal{S}^{\prime}\right|=64$.

To solve the first problem a service architecture, namely Service Oriented Optical Network (SOON) architecture, based on distributed signalling among designated service nodes, has been designed to fulfil service requests issued by applications while masking the transport related implementation details from the abstract request of the service. To solve the second problem, a techno-economic algorithm is proposed to help MPLS routers take the decision whether to switch traffic flows (Label Switched Paths or LSPs) optically or electronically.

\section{A. Advanced Connectivity Service Provisioning in GMPLS Networks}

The SOON architecture consists of a GMPLS-enabled transport network on top of which is added a new functional layer, called Service Plane (SP) [33]. The SP translates a network service request issued by an Application Entity (AE) into a set of technology-dependent directives to the network devices. The SP is composed by one Centralized Service Element (CSE) and a number of Distributed Service Elements (DSEs). The CSE performs AE identification and authorizes the relevant service requests using the information stored in its Service Level Agreement (SLA) database. The DSEs process network service requests via a User to Service Interface (USI), and interact with the other DSEs to perform the necessary technology-specific network setting into the controlled edge network nodes via a User to Network Interface (UNI).

An implementation of DSE and CSE was realized in Java to validate the SOON architecture Fig. 8 . The case study presented here refers to the on-demand set-up of L2/L3 VPN with QoS assurance across a MPLS network.

First of all the signalling delay was evaluated using two PCs connected to the Customer Edge (CE) routers. Each PC runs an instance of VLC media player; the former configured as a Video Server transmitting DVD video, the latter as Video Client. The time needed by the SP to fulfil the service request (i.e., overall service provisioning time) is about $13 \mathrm{~s}$. This time is not significantly affected by the number of routers involved, since the SP configures them in parallel and approximately at the same time. In particular, the processing time of the SP is about $1.8 \mathrm{~s}$ and 


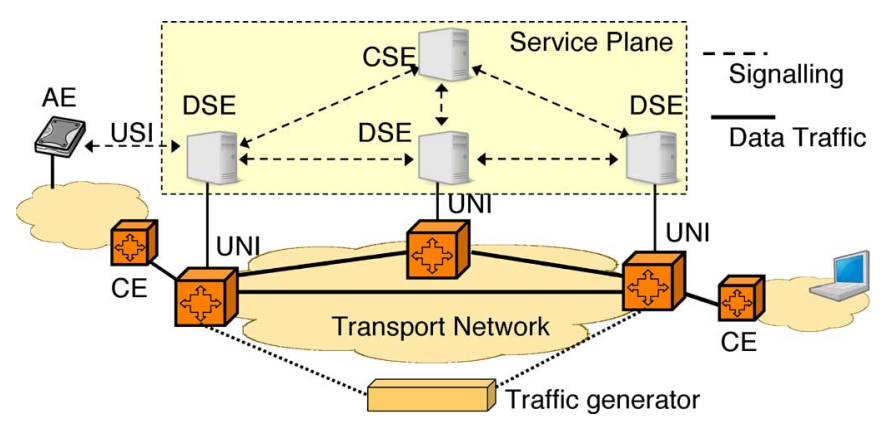

Fig. 8. SOON testbed for on-demand VPN set-up and QoS validation.

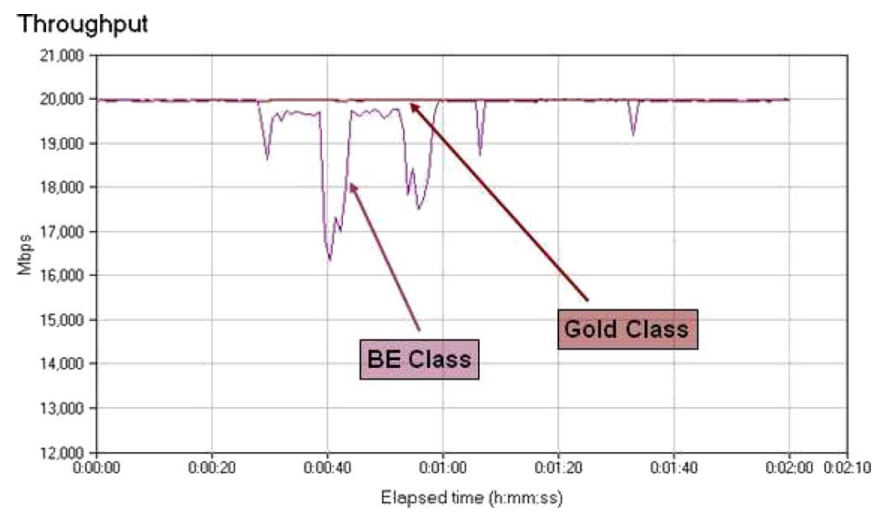

Fig. 9. Throughput versus elapsed time for HD streaming video and best effort traffic.

the time needed by the router to elaborate the UNI commands is about $2.5 \mathrm{~s} \mathrm{[34]}$.

To validate also the QoS QoS capabilities of proposed solution best effort traffic, mapped to the DiffServ BE class, and gold traffic, mapped to the Diffserv Expedited Forward (EF) class, were mixed. Specifically, the link under test was loaded with a High Definition (HD) Video Stream tagged in Gold Class (about $20 \mathrm{Mb} / \mathrm{s}$ ). Then traffic congestion was produced by using a traffic generator and applying a load equal to 980 Mbps (leading to an overall traffic load equal to $100 \%$ ) marked in BE Class. The objective throughput and the user perceived quality were evaluated. As desired the Gold class steadily maintained the throughput (Fig. 9), permitting and excellent video quality, thus proving that the QoS requirements of the video traffic were well satisfied [35].

\section{B. Multilayer Switching Algorithm for an All-Optical Router}

When the switching nodes have multiple switching alternatives (electronic, optical fibre based, optical wavelength based, optical packet based etc.) an important question to answer is how to map the traffic flows on the switching layers. This activity proposed a solution based upon a set of suitably chosen metrics [36].

First of all is computed a loss function that is used to quantify the effect of congestion. Based on the loss function is computed the Bayes risk [37] which is the expectation of the loss function as a function of the cost of queuing for a certain amount of time.

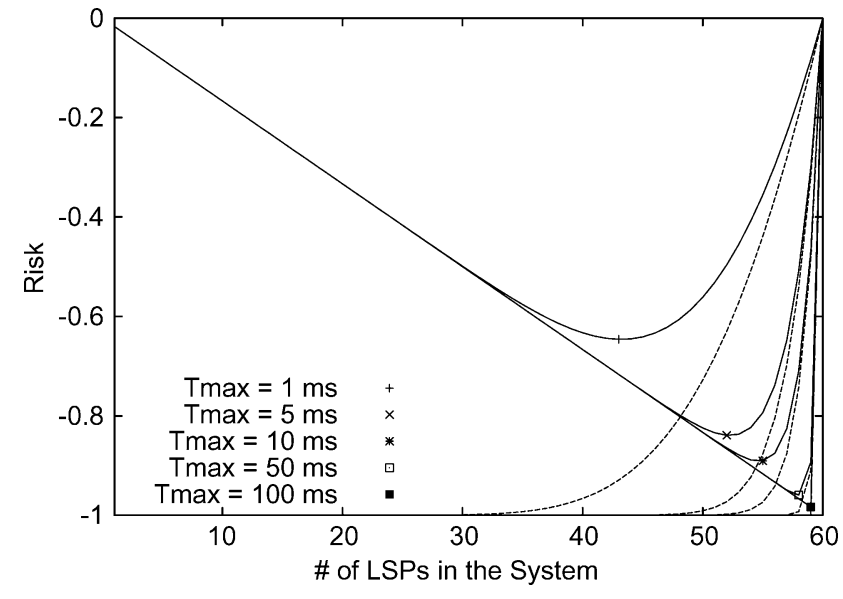

Fig. 10. Optimal decisions for several $T_{\max }$ values assuming hard real-time utility function (Dashed line $=$ Utility).

The goal of the algorithm is to obtain the optimal decision for the routing of the LSPs such that the Bayes risk is minimum.

This is combined with the definition of a set of utility functions that measure the QoS experienced (in terms of queuing delay) by the electronically-switched packets. Three utility functions are proposed.

- Mean utility: Computes the mean delay of the LSPs in the electronic domain.

- Hard-real time utility: Evaluates the probability that the delay in the router queue is lower than a given $T_{\max }$ threshold.

- Elastic utility: Assesses the gradual degradation of elastic services.

Finally a metric is introduced to quantify the relative cost of optical switching with respect to electronic switching $\left(R_{\text {cost }}\right)$. We have considered a linear cost approach, that evaluates the ratio at which the optical cost increases with respect to the electronic cost.

Fig. 10 shows the risk function and the utility function (dashed line) when QoS constraints changes assuming the hard-real time utility. The optimal decision is given by the mark in all curves. For instance the optimal decision for $T_{\max }=1 \mathrm{~ms}$ is 43 ; i.e., $43 \mathrm{LSPs}$ out of 60 are switched using the electronic layer.

Fig. 11 shows the impact of $R_{\text {cost }}$ using mean utility function, when the number of incoming LSPs in the system increases. $R_{\text {cost }}$ refers to the relative cost of optical switching with respect to electronic switching. When optical switching becomes expensive (large values of $R_{\text {cost }}$ ), less LSPs are switched optically.

Regarding the characteristics of the traffic sources, the results obtained show that the mean or variance of the incoming flows influence the decisor behaviour and helps to change the decision based on the traffic features [36]. However, when optical switching becomes too expensive, the $R_{\text {cost }}$ is critical in the optimal decision, thus cancelling any influence of the other parameters. In this light, the network operator has to decide where the optimal decision lies, trading off the $R_{\text {cost }}$ parameter and the incoming traffic parameters. 


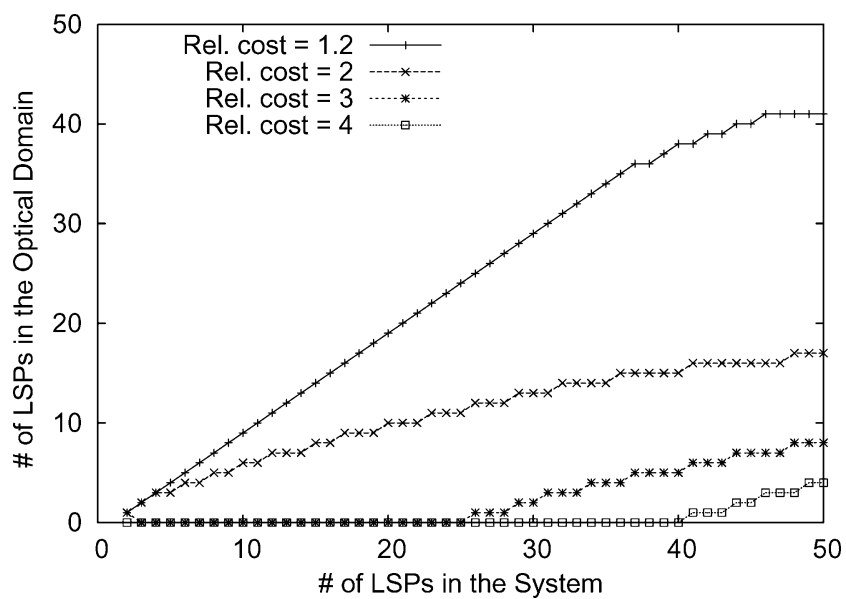

Fig. 11. Optimal decisions when $R_{\text {cost }}$ varies using the mean utility function.

\section{CONCLUSION}

This paper reported a summary of the joint research activities on core optical networks within the e-Photon-ONe+ project. This experience showed that it is possible to leverage on the integration of different expertises to tackle new problems that would prove difficult to be addressed by a single research group.

In the various JAs, the research approach was efficient and focused on selected aspects, aiming at providing guidelines and solutions that may be of help to the network engineer in the medium/long term.

We believe this paper shows that optical core networks are still a lively research topic and, most of all, that solutions and evolutionary paths towards their full implementation exist.

\section{REFERENCES}

[1] FP6 Instruments Task Force, Provisions for Implementing Networks of Excellence European Commission, Edition, May 12, 2003 [Online]. Available: http://cordis.europa.eu/fp6/instr_noe.htm

[2] F. Callegati, F. Neri, and P. Poggiolini, "The network of excellence e-Photon/ONe on optical networks," presented at the OFC, Anaheim, CA, Mar. 2006.

[3] [Online]. Available: http://networks.cs.ucdavis.edu/ mukherje/USEU-wksp-June05.html

[4] F. Callegati et al., "Research on optical core networks in the e-Photon/ONe network of excellence," presented at the IEEE Infocom, Barcelona, Spain, Apr. 2006.

[5] M. Poikselka et al., The IMS: IP Multimedia Concepts and Services, 2nd ed. New York: Wiley, 2006.

[6] K. Knightson et al., "NGN architecture: Generic principles functional architecture, and implementation," IEEE Commun. Mag., vol. 43, no. 10, 2005.

[7] J. Aracil et al., "Research in optical burst switching within the e-Photon/ONe network of excellence," Opt. Switching Netw., vol. 4, no. $1,2007$.

[8] P. Soproni, M. Perenyi, and T. Cinkler, "Grooming-enhanced multicast in multilayer networks," presented at the ONDM, Athens, May 2007.

[9] M. Perenyi et al., "Regular reconfiguration of light-trees in multilayer optical networks," presented at the ONDM, Athens, Greece, March 2008.

[10] N. Sengezer et al., "A comparative study of single-layer and multilayer traffic engineering approaches on transparent optical networks," presented at the 9th Int. Conf. Transparent Optical Networks, Rome, Italy, Jul. 2007.

[11] S. Spadaro et al., "The CARISMA ASON/GMPLS network: Overview and open issues," presented at the ICTON, Rome, Italy, Jul. 2007.
[12] P. Ghobril, "Analytical model for dynamic waveband switching," presented at the ONDM, Catalonia, Spain, Mar. 2008.

[13] P. Ghobril, "A novel analytical model for dynamic waveband switching," Optical Switching and Networking, Mar. 2009, accepted for publication.

[14] B. Quinn and K. Almeroth, IP Multicast Applications: Challenges and Solutions, IETF RFC 3170, Sep. 2001.

[15] L. H. Sahasrabuddhe and B. Mukherjee, "Light trees: Optical multicasting for improved performance in wavelength routed networks," IEEE Commun. Mag., vol. 37, no. 2, pp. 67-73, Feb. 1999.

[16] N. K. Singhal, L. H. Sahasrabuddhe, and B. Mukherjee, "Optimal multicasting of multiple light-trees of different bandwidth granularities in a WDM mesh network with sparse splitting capabilities," IEEE/ACM Trans. Networking, vol. 14, no. 5, pp. 1104-1117, 2006.

[17] R. Inkret et al., Advanced Infrastructure for Photonic Networks: Extended Final Report of COST Action 266 Faculty Elect. Eng. Comput., Univ. Zagreb, 2003.

[18] J. Comellas et al., "Integrated IP/WDM routing in GMPLS-based optical networks," IEEE Network, pp. 22-27, 2003.

[19] M. C. Necker, C. M. Gauger, and S. Bodamer, "A new efficient integrated routing scheme for $\mathrm{sdh} /$ sonet-wdm multilayer networks," presented at the OFC, Atlanta, GA, 2003.

[20] J. Szigeti et al., "p-Cycle protection in multi-domain environment," Photonic Netw. Commun., Sep. 2008.

[21] D. Staessens et al., "Computation of high availability connections in multidomain IP-over-WDM networks," presented at the Infocom, .

[22] D. Staessens et al., "Enabling high availability over multiple optical networks," IEEE Commun. Mag., vol. 46, pp. 120-126, Jun. 2008.

[23] M. Tornatore et al., "Effects of outdated control information in control-plane-enabled optical networks with path protection," IEEE/OSA J. Opt. Commun. Netw., Jul. 2009, to appear.

[24] P. Castoldi et al., "Centralized vs. distributed approaches for encompassing physical impairments in transparent optical networks," presented at the ONDM, Athens, Greece, May 2007.

[25] F. Cugini et al., "GMPLS extensions to encompass shared regenerators in transparent optical networks," in Proc. ECOC, Berlin, Germany, Sep. 2007.

[26] R. Martinez et al., "Experimental shared path protection in distributed all-optical GMPLS-based networks," presented at the DRCN, La Rochelle, France, Oct. 2007.

[27] B. Ramamurthy et al., "Impact of transmission impairments on the teletraffic performance of wavelength-routed optical networks," IEEE J. Lightw. Technol., vol. 17, no. 10, Oct. 1999.

[28] F. Callegati et al., "Congestion resolution in optical burst/packet switching with limited wavelength conversion," presented at the IEEE Globecom, San Francisco, CA, Nov. 2006.

[29] F. Callegati, W. Cerroni, and G. S. Pavani, "Key parameters for contention resolution in multi-fiber optical burst/packet switching node," presented at the Broadnets, Raleigh, NC, Sep. 2007.

[30] S. Yao, B. Mukherjee, S. J. B. Yoo, and S. Dixit, "A unified study of contention-resolution schemes in optical packet-switched networks," IEEE J. Lightw. Technol., vol. 21, no. 3, pp. 672-683, Mar. 2003.

[31] P. Pavon-Marino, F. J. Gonzalez-Castano, and J. Garcia-Haro, "RoundRobin wavelength assignment: A new packet sequence criterion in optical packet switching SCWP networks," Eur. Trans. Telecommun., vol. 17, no. 4, pp. 451-459, 2006.

[32] J. Veiga-Gontan, P. Pavon-Marino, and J. Garcia-Haro, "Reduction of packet order performance penalty in IBWR switches," presented at the 9th Int. Conf. Transparent Optical Networks, Sousse, Tunisia, Dec. 6-9, 2007.

[33] B. Martini, F. Baroncelli, and P. Castoldi, "A novel service oriented framework for automatically switched transport network," presented at the Integrated Network Management (IM), Nice, France, May 2005.

[34] F. Baroncelli et al., "A distributed signaling for the provisioning of ondemand VPN services in transport networks," presented at the Integrated Network Management (IM), Munich, Germany, May 2007.

[35] B. Martini et al., "Dynamic QoS control based on VPLS in service oriented transport networks," in Proc. ICTON, Jun. 22-26, 2008, vol. 1 , pp. $29-32$

[36] V. López et al., "A bayesian decision theory approach for the technoeconomic analysis of an all-optical router (extended version)," Comput. Netw., vol. 52, no. 1, 2008. 
[37] S. French and D. Ríos Insúa, Statistical Decision Theory. Oxford, U.K.: Oxford Univ. Press, 2000.

Franco Callegati (M'98) received the M.S. and Ph.D. degrees in electrical engineering in 1989 and 1992 from the University of Bologna, Italy.

He currently serves as an Associate Professor at the University of Bologna. He was a research scientist at the Teletraffic Research Centre of the University of Adelaide, Australia; Fondazione U. Bordoni, Italy; and the University of Texas at Dallas. His research interests are in the field of teletraffic modeling and performance evaluation of telecommunication networks. He has been working in the field of all optical networking since 1994 with particular reference to network architectures and performance evaluation for optical burst and packet switching. He has participated in several research project on optical networking at the national and international level, such as ACTS KEOPS, IST DAVID, and IST Ephoton/ONe, often coordinating work packages and research activities.

Filippo Cugini received the Laurea degree in telecommunication engineering from the University of Parma, Italy.

Since 2001, he has been a research engineer at the SSSUP/CNIT National Laboratory of Photonics Networks, Pisa, Italy. His main research interests include MPLS and GMPLS protocols and architectures, survivability in IP over WDM networks, and traffic engineering in grid networking.

Paul Ghobril received the electrical and electronics engineering degree in 1994 from the Lebanese University and the Ph.D. degree in computer science and networking in 2005 from ENST-Paris (now Télécom ParisTech), France.

He worked from 1994 to 2001 on designing electronic boards while teaching in major Lebanese universities. He joined France Telecom R\&D in 2006 as a postdoctorate researcher. In 2008, he created Envergus Sarl, a software and hardware engineering company located in Lannion, France. His main research interest is in modeling, simulation, and optimization of optical network technologies.

Sebastian Gunreben received the Dipl.-Ing. degree in mechatronics in 2004 from the University of Stuttgart, Germany.

Since then, he has been with the Institute of Communication Networks and Computer Engineering (IKR) at the University of Stuttgart where he works on traffic engineering for IP-over-WDM networks in several national and European projects. He focuses on control plane aspects of multi-layer networks as well as on the formal description of out-of-sequence packet arrivals.

Víctor López (S’08) received the M.Sc. degree in telecommunications engineering with Honors from the Universidad de Alcalá in 2005 and the PhD. degree in computer science and telecommunications engineering with Honors from the Universidad Autonoma de Madrid in 2009.

In 2004, he joined Telefonica I+D (R\&D) where he was a researcher in next generation networks for metro, core, and access. During this period, he participated in several European Union projects (NOBEL, MUSE, MUPBED) focused on those topics. In 2006, he joined the Networking Research Group of Universidad Autónoma de Madrid as a researcher in the ePhoton/One+ Network of Excellence. His research interests are on the analysis and characterization of services, design, and performance evaluation of traffic monitoring equipment, and the integration of Internet services over WDM networks, mainly OBS solutions and IP over WDM architectures.

Barbara Martini (M'06) received the M.S. degree in electronic engineering in 1999 from the University of Florence, Italy.

She joined Italtel as a Hardware Engineer working on network device drivers design and TCP/IP stack protocols and Marconi Communications in the summer of 2000 as Software Engineer involved in network management software design in DWDM equipments. Since 2003, she has been a Research Engineer at the CNIT National Laboratory of Photonics Networks located in Pisa, Italy. Her main research interests include network management system design, GMPLS optical control planes, and service platform architectures in next generation networks.
Pablo Pavon-Mariño (M'03) received the telecommunication engineering degree in telecommunications in 1999 from the University of Vigo (UVIGO), Spain, and the Ph.D. degree from the Technical University of Cartagena (UPCT) in 2004.

In 2000, he joined the UPCT, where he is an Associate Professor in the Department of Information Technologies and Communications. His research interests include performance evaluation, planning, and optimization of communication networks.

Marcell Perényi received the M.Sc. degree in computer science from the Budapest University of Technology and Economics (BUTE), Hungary, in 2005. He is currently pursuing the Ph.D. degree in the Department of Telecommunication and Media Informatics.

He has participated in several research projects supported by the EU and the Hungarian government. His research activities include simulation, algorithmic optimization, and planning of optical networks, as well as identification and analysis of traffic in IP networks, especially P2P, VoIP, and other multimedia applications. He has experience in planning, optimization, and maintenance of database systems, web services, and Microsoft infrastructures.

Namik Sengezer (S'04) received the B.S. and M.S. degrees in electrical engineering from Bilkent University, Turkey, in 2002 and 2004, where he is currently working toward the Ph.D. degree.

His research interests include design and planning of optical networks and traffic engineering.

Dimitri Staessens received the M.S. degree in numerical computer science in 2004 from Ghent University, Belgium.

He is now a member of the Department of Information technology, Ghent University, and the Interdisciplinary Institute for BroadBand Technology (IBBT). His research focuses on the design and evaluation of the next generation of communication networks, and he is currently involved in the European projects BONE and DICONET.

János Szigeti received the M.Sc. degree from the Budapest University of Technology and Economics (BME), Hungary, in 2002, where he is currently working toward the Ph.D. degree in the Department of Telecommunications and Media Informatics (TMIT).

His research interests focus on routing, design, configuration, dimensioning, and resilience of IP, MPLS, ATM, ngSDH, and particularly of WR-DWDMbased multilayer multidomain networks.

Massimo Tornatore (S'03-M'07) received the degree (Laurea) in telecommunications engineering in 2001 and the Ph.D. degree in information engineering in May 2006 from the Politecnico di Milano, Milan, Italy.

During his Ph.D. course, he worked in collaboration with Pirelli Telecom Systems and Telecom Italia Labs, and he was a visiting Ph.D. student in the Networks Lab of the University of California, Davis, and in CTTC (Technological Telecommunication Center of Catalunia), Barcelona, Spain. He is currently a postdoctorate researcher in the Department of Computer Science, University of California, Davis. He is the author of about 50 conference and journal papers and his research interests include design, protection strategies, traffic grooming in optical WDM networks, and group communication security.

Dr. Tornatore was a corecipient of Best Paper Award from IEEE ANTS 2008 and the Optical Networks Symposium in IEEE GlobeCom 2008. 